\title{
Innovative integrative tools and platforms. Key results of the PREPARE European Project
}

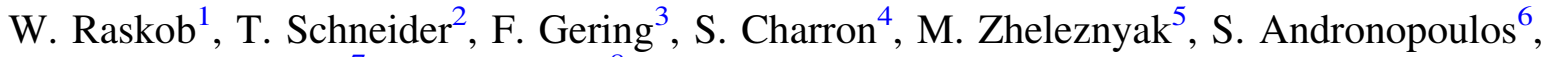 \\ G. Heriard-Dubreuil ${ }^{7}$ and J. Camps ${ }^{8}$ \\ ${ }^{1}$ Karlsruher Institut für Technologie (KIT), Eggenstein-Leopoldshafen, Hermann-von-Helmholtz-Platz 1, Germany. \\ 2 Nuclear Protection Evaluation Centre (CEPN), Fontenay-aux-Roses, France. \\ ${ }^{3}$ Federal Office for Radiation Protection (BfS), Neuherberg, Germany. \\ ${ }^{4}$ Nuclear Radiation Protection and Safety Institute (IRSN), Paris, France. \\ ${ }^{5}$ Ukrainian Center of Environmental and Water Projects (UCEWP), Kiev, Ukraine. \\ 6 National Center for Scientific Research "DEMOKRITOS", Aghia Paraskevi, Greece. \\ ${ }^{7}$ MUTADIS, Paris, France. \\ ${ }^{8}$ Belgian Nuclear Research Centre, SCK•CEN, Mol, Belgium.
}

\begin{abstract}
The European project PREPARE (Innovative integrated tools and platforms for radiological emergency preparedness and post-accident response in Europe) aims at closing gaps that have been identified in nuclear and radiological preparedness following the first evaluation of the Fukushima disaster. It started February 2013 and ended January 2016. Fourty-six partners from Europe and Japan, among them universities, national research centres, operational emergency management centres and non-governmental organisations, performed research to close gaps identified following the Fukushima disaster. Among others, the project addressed the review of existing operational procedures for dealing with long lasting releases, cross border problems in radiation monitoring and food safety and further development of missing functionalities in decision support systems ranging from improved source term estimation and dispersion modelling to the inclusion of hydrological pathways for European water bodies. In addition, a so called Analytical Platform was developed exploring the scientific and operational means to improve information collection, information exchange and the evaluation of such types of disasters. The tools developed within the project will be integrated into the two decision support systems ARGOS and RODOS.
\end{abstract}

Keywords: European project PREPARE / decision support / Fukushima / dispersion / aquatic / communication / trade

\section{Introduction}

The European project PREPARE (Innovative integrated tools and platforms for radiological emergency preparedness and post-accident response in Europe) aimed at closing these gaps that have been identified in nuclear and radiological preparedness following the first evaluation of the Fukushima disaster. Gaps identified comprised the question if existing EPR (emergency preparedness and response) procedures are fit for dealing with long lasting releases, how to deal with cross border monitoring and food safety and how to improve source term estimation and dispersion modelling as well as improve the modelling of hydrological pathways for European water bodies. Further to these very technical topics, trustworthiness of communication and the information collection and dissemination from one central location were part of the issues indicated by the review.

\section{Work program}

The PREPARE project was subdivided into seven research and one management activity (see Figure 1). The management activity concentrates in the operation of the project. The research work packages include also activities related to training and dissemination which was of high importance to achieve the objectives to harmonise response in Europe.

- Development of operational procedures for long lasting releases.

- Development of a so-called "Analytical Platform" with the objective to serve as a focal point for the collection of and analysis of information from any nuclear or radiological event, particularly regarding the consequences and any further developments.

- Development of recommendations related to quality control and management of contaminated goods which are applicable to whole Europe, taking into account the viewpoint of all relevant stakeholders (e.g. producers, retailers, consumers and administrations at national and regional levels).

- Improvement of atmospheric components of decision support systems such as ARGOS and RODOS in particular the estimation of a potential source term based on a combination of atmospheric dispersion calculations and 


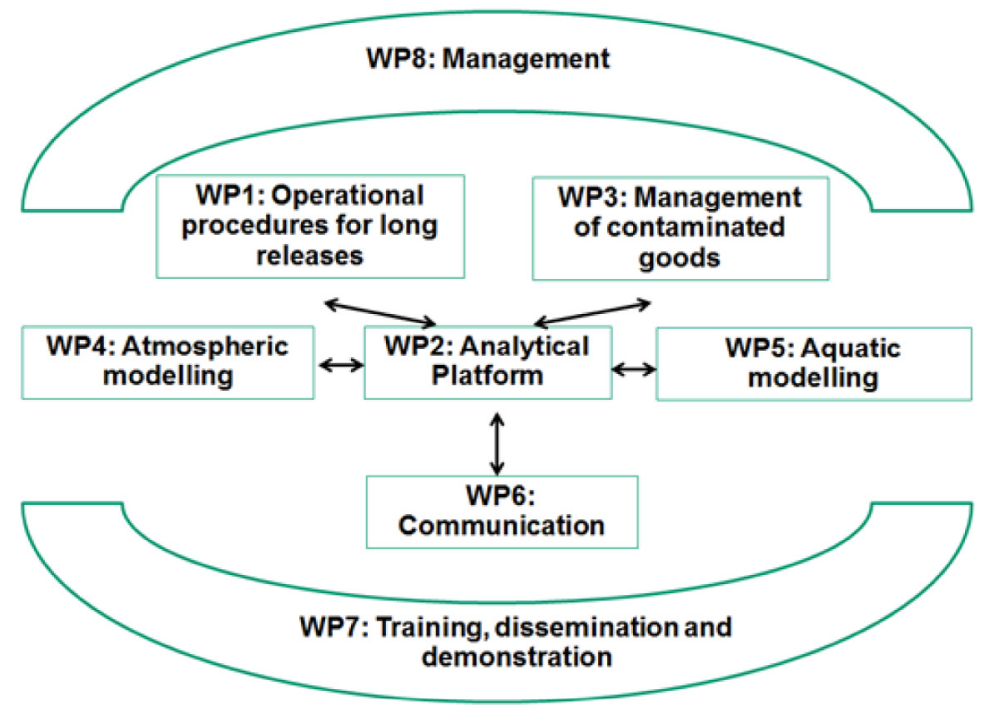

Figure 1. Dependencies of the PREPARE work packages.

monitoring data around a power plant and the physicochemical properties of radionuclides emitted to the atmosphere.

- Improvement to aquatic aspects of decision support systems by integrating state of the art aquatic models into the RODOS DSS and couple them with countermeasure simulation models.

- Investigate the conditions and means for relevant, reliable and trustworthy information to be made available to the public at the appropriate time and according to its needs, both during the nuclear emergency as well as in the postemergency phases.

- Basic training courses in the field of nuclear and radiological emergency and recovery planning and response and the organization of specific exercises related to transport accidents and large scale cross border contamination monitoring should be organized.

\section{Results}

After three years of research, the following brief summary lists the project achievements within the seven work packages:

- Operational procedures for long lasting releases: Following the Fukushima Daiichi accident a review of existing EPR procedures for long lasting releases and identification of possible needs for improvements by performing scenario calculations has been performed at a European level. Suggestions for improvements have been formulated, however, the overall performance of existing procedures was in most cases appropriate.

- Platform for information collection and exchange: The so-called Analytical Platform (AP) for information exchange in times of nuclear or radiological crisis allows discussion between institutional and non-institutional experts on an expert-level and to widespread congruent information on the current situation to the public including mass media. To support the expert in the analysis, a knowledge database has been developed, which contains historic events and scenarios, their propagation with time, and applied countermeasures and using the methodology Case-based reasoning (CBR) to find solutions for events that are not part of the database. Historic cases from Chernobyl and scenarios prepared with the RODOS system, approved by experts, populates the data base. The usage of this tool will be further explored with interested organisations in Europe.

- Management of contaminated goods: Stakeholder panels have been prepared and panels have had meetings in Belgium, Finland, France, Switzerland, Greece, Ireland, Netherlands, Norway, Portugal, Spain and United Kingdom to review existing guidance and to identify areas for improvement. It helps in designing and strengthening the preparedness plans for post-accidental situations at the national and local levels in European countries. A report summarising the results of all panels was prepared.

- Improvement to terrestrial aspects of decision support systems: The work here concentrated on the improvement of the atmospheric dispersion models implemented in the two DSS ARGOS and RODOS. Two methods for sourceterm estimation have been developed and implemented. A further important work activity was the improvement of the models with respect to the treatment of particles. And finally, models have been improved for long-range transport.

- Improvement to aquatic aspects of decision support systems: The aquatic model chain of the RODOS system has been improved in several aspects. The long-term watershed model MOIRA was integrated into the RODOS system. The global ocean model MyOcean (MyOcean, 2016) was linked to RODOS allowing to use the simulations of this model as boundary conditions for the simulation of radionuclide dispersion in RODOS. The simulation models were extensively tested for the Fukushima accident.

- Communication with the public: The overall objective of the work package was to investigate the conditions and 
means for relevant, reliable and trustworthy information to be made available to the public at the appropriate time and according to its needs, both during the nuclear emergency as well as in the post-emergency phases. Here traditional and social media have been studied. Important to note is also the interaction with the Analytical Platform activity exploring the involvement of experts and the public in the Platform and what are the rules and conditions that governmental and non-institutional experts interact with each other.

- Training, exercises and dissemination: Training and exercising was an important aspect of any RTD development and therefore treated as a separate work package. Training courses for early and late phase aspects in a nuclear or radiological emergency were prepared and conducted. A transboundary monitoring exercise was conducted. Training courses were also prepared and performed for the products developed within the project, in particular for the Analytical Platform. The results were disseminated at the final workshop held in Bratislava, January 2016. Nearly 120 participants from project partners, interested experts and international organisations participated in the event.

\section{Discussion and conclusions}

As a conclusion one can state, that PREPARE improved tools and methods in topics such as long lasting releases, source term estimation, model improvements, knowledge gathering and exchange of trustworthy information. It was clearly driven from the observations during and after the Fukushima incident. PREPARE is a step forward in harmonisation of emergency management and rehabilitation preparedness in Europe as it provides tools and methodologies which are either used in national organisation and implemented in decisions support systems such as ARGOS and RODOS, but also involving non-governmental stakeholders to gather and disseminate trustworthy information.

Acknowledgement. The research leading to these results has received funding from the European Atomic Energy Community Seventh Framework Programme FP7/2012-2013 under grant agreement 323287.

\section{Reference}

http://marine.copernicus.eu/ (accessed 30.03.2016)

Cite this article as: W. Raskob, T. Schneider, F. Gering, S. Charron, M. Zheleznyak, S. Andronopoulos, G. Heriard-Dubreuil, J. Camps. Innovative integrative tools and platforms: Key results of the PREPARE European Project. Radioprotection 51(HS2), S59-S61 (2016). 